\title{
CARACTERIZACIÓN DE LA ACCIÓN PEDAGÓGICA DE DOCENTES
}

\author{
CARACTERIZAÇÃO DA AÇÃO PEDAGÓGICA DOS PROFESSORES \\ CHARACTERIZATION OF THE PEDAGOGICAL ACTION OF TEACHERS
}

\author{
Adriana del Rosario Pineda Robayo ${ }^{1}$ \\ Secretaría de Educación departamento del Atlántico
}

Derlys Aminta Villadiego²

Universidad de la Costa CUC

\section{Resumen}

El objetivo del estudio es presentar un constructo que mida de forma general las características de la acción pedagógica de los profesores de Educación Inicial y primeros grados de Básica Primaria, en el sector público del Departamento del Atlántico, Colombia; enfocados en planeación, estrategias didácticas y ambientes educativos. Se realizó una prueba de consistencia interna, un análisis factorial exploratorio y un análisis factorial confirmatorio a la muestra piloto y final, de 135 y 130 docentes respectivamente, implementando SPSS y AMOS. Se comprobó que el cuestionario es confiable, para la prueba piloto y para la prueba definitiva, con un alfa de Cronbach de 0.782 y 0.756 respectivamente, pero no es válido tal como lo sustentan el estadístico Chi cuadrado y los índices de ajuste GFI, CFI, AGFI y RMSEA. Esta investigación se consolida como una guía para evaluar la práctica docente y facilitar su desarrollo profesional.

Palabras clave: Educación; Cuestionario; Coeficiente; Correlación y Validación.

\section{Resumo}

O objetivo do estudo é apresentar um construto que mede de maneira geral as características da ação pedagógica dos professores de Educação Infantil e do primeiro ano do Ensino Fundamental, no setor público do Departamento de Atlântico, Colômbia; focada em planejamento, estratégias didáticas e ambientes educacionais. Um teste de consistência interna, uma análise fatorial exploratória e uma análise fatorial confirmatória foram realizados na amostra piloto e na amostra final, de 135 e 130 professores, respectivamente, implementando o SPSS e o AMOS. O questionário foi considerado confiável, para o teste piloto e para o teste definitivo, com um alfa de Cronbach de 0,782 e 0,756,

1 PhD en Ciencias de la Educación. Docente Secretaría de Educación departamento del Atlántico. Colombia. adripineda10@hotmail.com.

2 Magíster en Estadística Aplicada. Docente Universidad de la Costa CUC. Barranquilla-Colombia. dvilladi3@cuc.edu.co. 
respectivamente, mas não é válido como suportado pela estatística do quadrado do Chi e pelos índices de ajuste GFI, CFI. AGFI e RMSEA. Esta pesquisa é consolidada como um guia para avaliar a prática de ensino e facilitar o desenvolvimento profissional.

Palavras-chave: Educação; Questionário; Quoeficiente; Correlação e Validação.

\section{Abstract}

The objective is to present a construct that measures in a general way the characteristic of the pedagogical action of the teachers of Initial Education and first grades of Primary Basic, in the public sector of the department of Atlántico, Colombia. The document focuses on planning, teaching strategies and educational environments. An internal consistency test, an exploratory factor analysis and a confirmatory factor analysis of the pilot and final sample of 135 and 130 teachers, respectively, were carried out with the help of SPSS and AMOS. The tests showed that the questionnaire is reliable for both the pilot test and the definitive test with a Cronbach alpha of 0.782 and 0.756 respectively, but not valid as supported by the Chi-square statistic and the GFI, CFI, AGFI and RMSEA adjustment rates. This research is the beginning of a guide to evaluate teaching practice and facilitate its professional development.

Keywords: Education; Questionnaire; Coefficient; Correlation and Validity.

\section{INTRODUCCIÓN}

El estudio de la relación entre la formación del docente y su práctica pedagógica en la cotidianidad del entorno educativo corresponde a una de las temáticas de mayor interés actualmente. La percepción de los docentes frente a las inconsistencias entre los saberes necesarios para resolver las tensiones presentes en las comunidades y con los niños con quienes desempeñan su labor y las oportunidades de desarrollo profesional que les ofrecen, en su mayoría teóricas y alejadas del contexto, hacen parte del foco desde el cual se desarrolla el presente estudio. En este sentido, emprender procesos de actualización docente en el contexto de la acción práctica, implica la posibilidad de acompañar a los docentes en la reflexión y metacognición constantes sobre los saberes necesarios para manejar estrategias flexibles, interactivas, experienciales y centradas en el desarrollo integral en los niños y las niñas.

El estudio plantea la importancia del apoyo constante al proceso de desarrollo profesional de los docentes de Educación Inicial, con experiencias y conocimientos pedagógicos y didácticos diferentes, como factor determinante en la calidad del servicio educativo. Este aspecto es proporcional al conocimiento de las características de la acción pedagógica del docente, en la posibilidad de generar procesos pertinentes y contextualizados de cualificación que lleven a la resignificación de las prácticas. Igualmente, el conocer y 
comprender la relación entre los procesos pedagógicos desarrollados por el docente y su capacidad para flexibilizar y utilizar diversas herramientas y estrategias pedagógicas, facilita el desarrollo de perspectivas que aseguren el compromiso con una Educación Inicial eficiente, significativa equilibrada en una sociedad culturalmente cambiante.

\section{CARACTERIZACIÓN DE LOS DOCENTES DE TRANSICIÓN Y PRIMER GRADO DE BÁSICA PRIMARIA}

El trascender de una perspectiva centrada en los conceptos de enseñanza, aprendizaje, transmisión de conocimientos, memoria y alejamiento de la vida real, hacia una visión en la cual el docente es actor principal de su desarrollo profesional, plantea la necesidad de acompañar y apoyar este proceso para que sea exitoso, duradero y significativo. En este sentido, desde diversas perspectivas teóricas se plantea la importancia de direccionar la formación continuada del docente hacia lo que denomina "formación para la acción", es decir hacia la capacidad de reinventar constantemente su quehacer a la luz de los retos que le propone la cotidianidad, (Aguerrondo, 2009).

Este acompañamiento, debería iniciar con el docente recién egresado de las aulas de pregrado y que se encuentra viviendo los primeros años de ejercicio profesional, quien se enfrenta a la complejidad de transferir las teorías y técnicas aprendidas en la universidad al contexto de realidad, donde debe solucionar múltiples situaciones. De acuerdo con Zanten et al. (2014) los nuevos docentes, manifiestan la dificultad para relacionar las ideas pedagógicas que les han transmitido en la universidad y las que ponen en práctica en el aula, teniendo en cuenta que la transferencia necesaria para que este proceso ocurra requiere de tiempo y el acompañamiento, a partir de la reflexión sobre la realidad del proceso educativo.

Este aspecto influye también en el proceso de construcción de su identidad como docente, ya que coincide con la habilidad para conciliar la distancia entre las perspectivas teóricas de formación y las realidades de su práctica. Igualmente, el fortalecimiento de su capacidad de desaprender modelos y prácticas provenientes de su propia experiencia como estudiante, expuesto a maneras diversas de ser docente, así como su fortaleza para conciliar las perspectivas presentes en los contextos donde se desempeñan con las ideas de transformación que traen como convicción.

El desarrollo profesional del docente, lo propone Rivilla (2015) como un estilo de vida permanente, disciplinado, enfocado a un aprendizaje activo desde las necesidades de la vida 
en el aula y en la institución, en el cual todos los profesores compartan saberes y necesidades, lo que dará lugar a la producción de nuevos saberes. La institución educativa se convierte en el centro del desarrollo profesional en la medida en que se fomente el desarrollo de actividades asociadas a la auto observación de la práctica y su análisis crítico y reflexivo.

Así, la acción pedagógica del docente es una construcción dinámica que se transforma progresivamente desde dos campos específicos: las experiencias personales, individuales asociadas a la forma particular de asumir los retos que presenta la cotidianidad, y el contexto social, cultural y educativo que influye sobre las percepciones e interpretaciones de la experiencia.

La conciencia crítica que respalda el abordaje de la complejidad inherente a este campo lleva a los investigadores a indagar acerca de las características de la acción pedagógica del docente de transición y primer grado de Básica Primaria del Departamento del Atlántico. Para esto se diseña un formato de caracterización desde el cual se estudian aspectos sustantivos como la planeación, estrategias didácticas utilizadas por los docentes y los ambientes educativos que propician. Para la construcción del instrumento de medición tal como lo indican Hernández et al. (2014): "se requiere reevaluar las variables de investigación, revisión enfocada de la literatura, identificación del dominio de las variables a medir y sus indicadores, utilización de un instrumento nuevo de medición ya elaborado o el desarrollo de uno nuevo, aplicación de una prueba piloto, revisión del instrumento, entrenamiento del personal que va administrar el instrumento, contar con las autorizaciones para la aplicación de representantes que estén implicadas en el estudio, administración del instrumento y la preparación de los datos para su análisis." (p. 209).

El objetivo del estudio es determinar la confiabilidad de consistencia interna por medio del alfa de Cronbach y la validez del constructo, a través de un análisis factorial exploratorio y un análisis factorial confirmatorio (Escobedo Portillo, Hernández Gómez, Estebané Ortega, \& Martínez Moreno, 2016) para crear un modelo que mida de forma general la acción pedagógica del docente de transición y primer grado de Básica Primaria (APEDOTP) de Instituciones Educativas públicas del Departamento del Atlántico.

Los paquetes estadísticos utilizados fueron SPSS versión 22 y AMOS versión 7 . 


\section{MÉTODO}

Al inicio se realizó una prueba piloto entre abril y junio del 2018 a una muestra probabilística e intencional a 135 docentes pertenecientes a 11 de los 23 municipios del Departamento del Atlántico (47.8\%), orientada a demostrar la pertinencia y eficacia del instrumento a través de un análisis de consistencia interna, un análisis factorial exploratorio y un análisis factorial confirmatorio. Se recolectaron 62 (45.9\%) formatos diligenciados por docentes del grado transición y $73(54.1 \%)$ contestados por profesores de primer grado, distribuidos así: 13 (9.6\%) del municipio de Baranoa (6 transición, 7 primero); 7 (5.2\%) de Campo de la Cruz (2 transición, 5 primero); 34 (25.2\%) de Galapa (15 transición, 19 primero); $10(7.4 \%)$ en Luruaco (5 transición, 5 primero); 19 (14.1\%) en Malambo (8 transición, 11 primero); 2 (1.5\%) en Polo Nuevo (transición); 1 (0.7\%) Puerto Colombia (transición); 13 (9.6\%) en Sabanagrande (8 transición, 5 primero); 26 (19.3\%) en Sabanalarga (12 transición, 14 primero); 4 (3\%) en Soledad ( 1 transición, 3 primero) y $6(4.4 \%)$ en Tubará ( 2 transición, 4 primero).

Los ítems de los componentes del constructo APEDOTP fue valorada por los docentes participantes en una escala tipo Likert, con cinco opciones de respuesta. Para la primera escala "Planeación y Métodos, las opciones de respuesta a cada ítem fueron: 1 (Nunca), 2 (Muy rara vez), 3 (En ocasiones), 4 (A menudo) y 5 (Muy a menudo). En el caso de la escala Ambientes el valor numérico es: 1(Totalmente en desacuerdo), 2 (Parcialmente en desacuerdo), 3 (Ni de acuerdo ni en desacuerdo), 4 (Parcialmente de acuerdo) y 5 (Totalmente de acuerdo), el cual expresan verbalmente el entendimiento de las preguntas y claridad en la información requerida. Luego de la revisión de la prueba piloto, se procedió a construir la versión definitiva del instrumento y se aplicó a 130 docentes, de Instituciones Educativas oficiales del Departamento del Atlántico. La recolección de la información se dio entre los meses de junio y julio de 2018 a una muestra probabilística e intencional de docentes que voluntariamente aceptaron participar diligenciando la encuesta. La muestra corresponde a 67 (51.5\%) del grado de transición y 63 (48.5\%) de primer grado, de los municipios Palmar de Varela 22 (16.9\%) (11 transición, 11 primero), Sabanalarga 97 (74.6\%) (52 transición, 45 primero) y Santo Tomás 11 (8.5\%) (4 transición, 7 primero).

La investigación se abordó desde el análisis de datos cuantitativo para evaluar la encuesta sobre las características de la acción pedagógica del docente de transición y primer 
grado de Básica Primaria (APEDOTP) de Instituciones Educativas públicas del Departamento del Atlántico. Para ello se usó el coeficiente de alfa de Cronbach, el análisis factorial exploratorio y el análisis factorial confirmatorio para comprobar la confiabilidad y validez del constructo.

\section{RESULTADOS}

Inicialmente, se consideró que las preguntas Q1 a Q10 hacen parte de la escala Planeación, los ítems Q11 a Q22 forman parte de la escala Métodos o Estrategias y las preguntas Q23 a Q30 conforman la escala Ambientes (ver Tabla I, Tabla II y Tabla III).

\section{Tabla I - Escala Planeación}

\begin{tabular}{l|l}
\hline \multicolumn{2}{c}{ ÍTEMS } \\
\hline Q1 & Lidero la organización de actividades de planeación \\
Q2 & Aporto ideas y propuestas orientadas a mejorar y/o innovar los procesos de planeación. \\
Q3 & La planificación de las actividades la realiza el coordinador(a) \\
Q4 & Las actividades que se planifican son flexibles o susceptibles de modificar. \\
Q5 & Realizo la planeación de manera individual. \\
Q6 & Realizo la planeación con mis compañeros de la IE. \\
Q7 & Organizo la planeación de las actividades teniendo en cuenta los problemas y necesidades \\
& del contexto. \\
Q8 & Tengo en cuenta, al planear, que los indicadores de logro sean coherentes con el momento \\
& evolutivo de los niños y sus necesidades. \\
Q9 & Diseño actividades que guarden coherencia con los proyectos institucionales. \\
Q10 & Tengo en cuenta un modelo pedagógico específico a la hora de planificar las actividades. \\
\hline
\end{tabular}

Fonte: Los propios autores

Tabla II - Métodos o Estrategias.

\begin{tabular}{l|l}
\hline & \multicolumn{1}{c}{ ÍTEMS } \\
\hline Q11 & Las actividades que realizo en el aula guardan coherencia con la propuesta institucional. \\
Q12 & Tiendo a controlar la disciplina de los niños y niñas para lograr el éxito de las actividades. \\
Q14 & En el salón propongo actividades que los niños realicen de manera autónoma. \\
Q15 & Las actividades que desarrollo con los niños integran diferentes dimensiones del desarrollo. \\
Q16 & Las actividades que realizo con los niños y niñas tienen en cuenta la dimensión corporal. \\
Q17 & Motivo permanentemente a los niños para que participen activamente en las actividades realizadas. \\
Q18 & La disciplina de los niños es importante en la consecución de los objetivos de las actividades. \\
Q19 & En el desarrollo de las actividades tengo en cuenta la lectura de cuentos y literatura infantil. \\
Q20 & Realizo actividades que incluyan el arte como mediador. \\
Q21 & Permito la espontánea y libre expresión de los niños y niñas. \\
Q22 & Propongo a los niños actividades retadoras para desplegar su creatividad y resolver problemas. \\
\hline
\end{tabular}

Fonte: Los propios autores 
Tabla III - Ambientes.

\begin{tabular}{l|l}
\hline \multicolumn{2}{c}{ ÍTEMS } \\
\hline Q23 & El espacio del que dispone es suficientemente ventilado. \\
Q24 & Las instalaciones de la institución presentan condiciones adecuadas de aseo y mantenimiento. \\
Q25 & Tiene un espacio adecuado e independiente del aula, donde los niños reciben alimentación. \\
Q26 & Las dimensiones del espacio en el que están los niños están de acuerdo con la normatividad. \\
Q27 & El espacio del que dispone tiene adecuada iluminación. \\
Q28 & Las instalaciones sanitarias son adecuadas y acordes con las necesidades de los niños (a escala) \\
Q29 & Los espacios pedagógicos se usan de acuerdo con la planeación y horarios establecidos. \\
Q30 & La dotación y recursos se usan de acuerdo con una planeación. \\
\hline
\end{tabular}

Fonte: Los propios autores

Ahora bien, de la prueba piloto se prescindió de tres formatos por no responder al $20 \%$ o más de los ítems de la encuesta; los datos perdidos fueron reemplazados con los promedios de sus respuestas correspondientes a los ítems de las escalas respectivas (Cohen, Cohen, West, \& Aiken, 2002; Schmidt \& Dantas, 2011).

Se realizó un análisis factorial exploratorio para determinar qué tan relacionados se encuentran los ítems en sus respectivos grupos (Pérez López, 2004; Herrero, 2010; Lloret, Ferreres, Hernández, \& Tomás, 2014). El valor del estadístico KMO es $0.77>0.5$, lo que indica una buena adecuación de la muestra a este tipo de análisis, el test de esfericidad de Bartlett con p-valor 0.000 , permite concluir que existe correlación significativa entre los ítems de cada dimensión (Magaña, Vásquez, \& Aguilar, 2017). La consistencia interna de todo el cuestionario se determinó calculando el coeficiente de alfa de Cronbach (Lizarbe, Guillén-, Aguinaga, \& Canga, 2016; Schmidt \& Dantas, 2011) cuyo valor es 0.766 que sobrepasa el valor mínimo aceptado por estudios (Cascaes da Silva et al., 2015).

Las comunalidades de los ítems Q3, Q4, Q5, Q6, Q11 y Q12 están muy alejadas de la unidad (Lacave, Molina, Fernández, \& Redondo, 2015), al ser retiradas del estudio el estadístico KMO y el coeficiente alfa de Cronbach de todo el cuestionario, obtuvieron puntuaciones de 0.787 y 0.782 , valores más altos que los resultados anteriores.

El análisis factorial proporcionó tres factores con autovalores mayores que 1, explicando el $46.785 \%$ de la variabilidad total del estudio. Para determinar los factores a los que pertenecen cada ítem, se realizó una rotación oblicua (oblimin directo) (Lloret et al., 2014). El primer factor está conformado por los ítems Q14, Q15, Q16, Q17, Q18, Q19, Q20, Q21, Q22 y explica el 24.707\%; el segundo factor está conformado por los ítems Q23, 
Q24,Q25,Q26,Q27,Q28,Q29,Q30 y explica el 14.874\% y el tercer factor explica el 7.204\% y está conformado por los ítems Q1,Q2,Q7,Q8,Q9,Q10 y Q13.

El alfa de Cronbach de las tres dimensiones son $0.861,0.81$ y 0.769 respectivamente, considerado adecuado por varios autores (Cascaes da Silva et al., 2015). Por otra parte, la correlación total de los elementos corregida de cada uno de los ítems, todos con valores mayores que 0.3 (ver Tabla IV), indican la no exclusión de ninguno de los ítems que conforman cada dimensión (Lizarbe et al., 2016).

Tabla IV - Correlación total de los elementos corregida.

\begin{tabular}{|c|c|c|c|c|c|c|c|c|}
\hline \multicolumn{3}{|c|}{ Factor 1} & \multicolumn{3}{|c|}{ Factor 2} & \multicolumn{3}{|c|}{ Factor 3} \\
\hline Ítems & $\begin{array}{c}\text { Correlación } \\
\text { total de } \\
\text { elementos } \\
\text { corregida }\end{array}$ & $\begin{array}{c}\text { Alfa de } \\
\text { Cronbach si el } \\
\text { elemento se ha } \\
\text { suprimido }\end{array}$ & Ítems & $\begin{array}{l}\text { Correlación } \\
\text { total de } \\
\text { elementos } \\
\text { corregida }\end{array}$ & $\begin{array}{c}\text { Alfa de } \\
\text { Cronbach si el } \\
\text { elemento se ha } \\
\text { suprimido }\end{array}$ & Ítems & $\begin{array}{c}\text { Correlación } \\
\text { total de } \\
\text { elementos } \\
\text { corregida }\end{array}$ & $\begin{array}{c}\text { Alfa de } \\
\text { Cronbach si el } \\
\text { elemento se ha } \\
\text { suprimido }\end{array}$ \\
\hline Q14 & 0.65 & 0.84 & Q23 & 0.52 & 0.79 & Q1 & 0.47 & 0.75 \\
\hline Q15 & 0.70 & 0.84 & Q24 & 0.45 & 0.80 & Q2 & 0.56 & 0.73 \\
\hline Q16 & 0.59 & 0.85 & Q25 & 0.45 & 0.80 & Q7 & 0.52 & 0.74 \\
\hline Q17 & 0.55 & 0.85 & Q26 & 0.52 & 0.79 & Q8 & 0.47 & 0.75 \\
\hline Q18 & 0.44 & 0.86 & Q27 & 0.60 & 0.78 & Q9 & 0.53 & 0.73 \\
\hline Q19 & 0.50 & 0.86 & Q28 & 0.55 & 0.79 & Q10 & 0.46 & 0.75 \\
\hline Q20 & 0.63 & 0.84 & Q29 & 0.52 & 0.79 & Q13 & 0.47 & 0.75 \\
\hline Q21 & 0.71 & 0.84 & Q30 & 0.60 & 0.78 & & & \\
\hline Q22 & 0.60 & 0.85 & & & & & & \\
\hline
\end{tabular}

Fonte: Los propios autores

Para comprobar la validez del cuestionario, se realizó un análisis factorial confirmatorio, los resultados mostraron (ver tabla V) que el modelo no presentó un buen ajuste (Pérez, Medrano, \& Rosas, 2013; Magaña et al., 2017).

Tabla V - Índice de ajuste de la prueba piloto

\begin{tabular}{ccccccc}
\hline$\chi^{2}$ & gl & p-valor & GFI & CFI & AGFI & RMSEA \\
\hline 381.396 & 249 & 0.000 & 0.823 & 0.865 & 0.786 & 0.064 \\
\hline
\end{tabular}

Fonte: Los propios autores

Posterior a la revisión de la prueba piloto se procedió a construir, en base a esta prueba, la versión definitiva del instrumento APEDOTP, dos de los 130 docentes que participaron en la prueba no contestaron el $20 \%$ o más de las preguntas, por tal motivo se excluyeron del 
estudio. Los datos perdidos se rellenaron con los promedios de sus respuestas correspondientes a los ítems de las escalas respectivas (Schmidt \& Dantas, 2011).

El análisis factorial exploratorio con $\mathrm{KMO}=0.773>0.5$ y test de esfericidad de Bartlett con p-valor 0.000 confirman la correlación de los ítems y la adecuación de los datos a un análisis factorial. La consistencia interna de todo el cuestionario se determinó hallando el coeficiente de alfa de Cronbach con valor 0.756.

Los tres factores explican el $50.669 \%$ de la variabilidad total, un poco más elevado que la prueba piloto, esto como resultado de las adecuaciones y correcciones que se hicieron a partir del análisis resultante de la aplicación de la prueba piloto. El primer factor explica el $27.764 \%$, el segundo factor explica el $15.535 \%$ y el tercer factor explica el $7.399 \%$, luego se realiza la rotación oblicua para determinar los ítems que conforman cada uno de los factores: el primer componente por Q1, Q7, Q8, Q9, Q10, Q13, Q14, Q15 y Q18, el segundo componente por Q23, Q24, Q5, Q26, Q27, Q28, Q29 y Q30 y el tercer componente por Q2, Q16, Q17, Q19, Q20, Q21 y Q22.

El coeficiente alfa de Cronbach de los tres factores son: $0.858,0.832$ y 0.833 respectivamente. Al analizar la correlación total de los elementos corregida de cada uno de los ítems, todos con valores mayores que 0.3 (ver Tabla VI), se procede a la no exclusión de ninguno de los ítems que conforman cada dimensión (Lloret et al., 2014; Lizarbe et al., 2016).

Tabla VI - Correlación total de los elementos corregida

\begin{tabular}{|c|c|c|c|c|c|c|c|c|}
\hline \multicolumn{3}{|c|}{ Factor 1} & \multicolumn{3}{|c|}{ Factor 2} & \multicolumn{3}{|c|}{ Factor 3} \\
\hline Ítems & $\begin{array}{l}\text { Correlación } \\
\text { total de } \\
\text { elementos } \\
\text { corregida }\end{array}$ & $\begin{array}{c}\text { Alfa de } \\
\text { Cronbach si el } \\
\text { elemento se } \\
\text { ha suprimido }\end{array}$ & Ítems & $\begin{array}{l}\text { Correlación } \\
\text { total de } \\
\text { elementos } \\
\text { corregida }\end{array}$ & $\begin{array}{c}\text { Alfa de } \\
\text { Cronbach si el } \\
\text { elemento se } \\
\text { ha suprimido }\end{array}$ & Ítems & $\begin{array}{l}\text { Correlación } \\
\text { total de } \\
\text { elementos } \\
\text { corregida }\end{array}$ & $\begin{array}{c}\text { Alfa de } \\
\text { Cronbach si el } \\
\text { elemento se } \\
\text { ha suprimido }\end{array}$ \\
\hline Q1 & 0.50 & 0.85 & Q23 & 0.61 & 0.81 & Q2 & 0.44 & 0.83 \\
\hline Q7 & 0.67 & 0.84 & Q24 & 0.69 & 0.80 & Q16 & 0.58 & 0.81 \\
\hline Q8 & 0.71 & 0.83 & Q25 & 0.40 & 0.84 & Q17 & 0.65 & 0.80 \\
\hline Q9 & 0.72 & 0.83 & Q26 & 0.67 & 0.80 & Q19 & 0.63 & 0.80 \\
\hline Q10 & 0.62 & 0.84 & Q27 & 0.65 & 0.80 & Q20 & 0.63 & 0.80 \\
\hline Q13 & 0.49 & 0.85 & Q28 & 0.40 & 0.83 & Q21 & 0.65 & 0.80 \\
\hline Q14 & 0.58 & 0.84 & Q29 & 0.51 & 0.82 & Q22 & 0.52 & 0.82 \\
\hline Q15 & 0.55 & 0.85 & Q30 & 0.59 & 0.81 & & & \\
\hline Q18 & 0.48 & 0.86 & & & & & & \\
\hline
\end{tabular}

Fonte: Los propios autores 
Los resultados del análisis factorial confirmatorio del modelo resultante del análisis factorial exploratorio, mostraron que el modelo de los tres factores que conforman el cuestionario no presentó un buen ajuste tal como lo muestran los valores estadísticos (ver Tabla VII).

Tabla VII - Índice de ajuste de la prueba definitiva

\begin{tabular}{ccccccc}
\hline$\chi^{2}$ & gl & p-valor & GFI & CFI & AGFI & RMSEA \\
\hline 499.5 & 242 & 0.000 & 0.767 & 0.809 & 0.711 & 0.092 \\
\hline
\end{tabular}

Fonte: Los propios autores

El análisis descriptivo de la información evidencia que más del $80 \%$ de los docentes tienden a contestar "A menudo" y "Muy a menudo" en el primer y tercer factor, y en el segundo factor del $40 \%$ al $65 \%$ de los participantes contestaron, "Totalmente de acuerdo" y "Parcialmente de acuerdo" (ver Tabla VIII, Tabla IX y Tabla X).

Tabla VIII - Porcentaje de las opciones de respuestas de los ítems del factor 1.

\begin{tabular}{|c|c|c|c|c|c|c|}
\hline Ítems & $\begin{array}{c}\text { Nunca } \\
(\%)\end{array}$ & $\begin{array}{c}\text { Muy rara vez } \\
(\%)\end{array}$ & $\begin{array}{c}\text { En ocasiones } \\
(\%)\end{array}$ & $\begin{array}{c}\text { A menudo } \\
(\%)\end{array}$ & $\begin{array}{c}\text { Muy a menudo } \\
(\%)\end{array}$ & $\begin{array}{c}\text { Sin respuestas } \\
(\%)\end{array}$ \\
\hline Q1 & & \multirow[t]{4}{*}{0.80} & 8.50 & 40.80 & 49.20 & 0.80 \\
\hline Q7 & & & 5.40 & 40.80 & 53.10 & 0.80 \\
\hline Q8 & & & 3.10 & 36.20 & 60.00 & 0.80 \\
\hline Q9 & & & 6.90 & 39.20 & 52.30 & 1.50 \\
\hline Q10 & & \multirow[t]{4}{*}{3.80} & 6.20 & 42.30 & 46.90 & 0.80 \\
\hline Q13 & & & 6.20 & 50.00 & 43.10 & 0.80 \\
\hline Q14 & & & 5.40 & 38.50 & 54.60 & 1.50 \\
\hline Q15 & & & 0.80 & 36.20 & 61.50 & 1.50 \\
\hline Q18 & & 0.80 & 14.60 & 44.60 & 39.20 & 0.80 \\
\hline
\end{tabular}

Fonte: Los propios autores 
Tabla IX - Porcentaje de las opciones de respuestas de los ítems del factor 2

\begin{tabular}{c|c|c|c|c|c|c}
\hline Ítems & $\begin{array}{c}\text { Totalmente } \\
\text { de acuerdo } \\
(\%)\end{array}$ & $\begin{array}{c}\text { Parcialmente } \\
\text { de acuerdo } \\
(\%)\end{array}$ & $\begin{array}{c}\text { Ni de acuerdo ni } \\
\text { en desacuerdo } \\
(\%)\end{array}$ & $\begin{array}{c}\text { Parcialmente } \\
\text { en desacuerdo } \\
(\%)\end{array}$ & $\begin{array}{c}\text { Totalmente en } \\
\text { desacuerdo } \\
(\%)\end{array}$ & $\begin{array}{c}\text { Sin respuestas } \\
(\%)\end{array}$ \\
\hline Q23 & 26.20 & 26.90 & 1.50 & 20.80 & 22.30 & 2.30 \\
Q24 & & & 5.40 & 40.80 & 53.10 & 0.80 \\
Q25 & & & 3.10 & 36.20 & 60.00 & 0.80 \\
Q26 & & & 6.90 & 39.20 & 52.30 & 1.50 \\
Q27 & & 3.80 & 6.20 & 42.30 & 46.90 & 0.80 \\
Q28 & & & 6.20 & 50.00 & 43.10 & 0.80 \\
Q29 & & & 5.40 & 38.50 & 54.60 & 1.50 \\
Q30 & & & 0.80 & 36.20 & 61.50 & 1.50 \\
\hline
\end{tabular}

Fonte: Los propios autores

Tabla $\mathbf{X}$ - Porcentaje de las opciones de respuestas de los ítems del factor 3 .

\begin{tabular}{c|c|c|c|c|c|c}
\hline Ítems & $\begin{array}{c}\text { Nunca } \\
(\%)\end{array}$ & $\begin{array}{c}\text { Muy rara vez } \\
(\%)\end{array}$ & $\begin{array}{c}\text { En ocasiones } \\
(\%)\end{array}$ & $\begin{array}{c}\text { A menudo } \\
(\%)\end{array}$ & $\begin{array}{c}\text { Muy a menudo } \\
(\%)\end{array}$ & $\begin{array}{c}\text { Sin respuestas } \\
(\%)\end{array}$ \\
\hline Q2 & & & 7.70 & 50.80 & 40.80 & 0.80 \\
Q16 & & 9.20 & 47.70 & 41.50 & 1.50 \\
Q17 & & 0.80 & 2.30 & 29.20 & 66.90 & 0.80 \\
Q19 & 0.80 & 3.80 & 36.90 & 57.70 & 0.80 \\
Q20 & & 0.80 & 5.40 & 46.20 & 45.40 & 2.30 \\
Q21 & 1.50 & & 33.10 & 63.10 & 2.30 \\
Q22 & 1.50 & 6.90 & 50.80 & 38.50 & 2.30 \\
\hline
\end{tabular}

Fonte: Los propios autores

\section{CONCLUSIÓN Y DISCUSIÓN}

Partiendo de la acción pedagógica del docente de transición y primer grado de Básica Primaria de las instituciones públicas del Departamento del Atlántico con la intencionalidad de medir la planeación, las estrategias didácticas utilizadas por los docentes y los ambientes educativos que propician, el estudio arrojó para la prueba piloto y la prueba definitiva, que el cuestionario es confiable de acuerdo al coeficiente alfa de Cronbach, medida también utilizada para demostrar la confiabilidad en investigaciones relacionadas con la educación en primaria y secundaria de (Sánchez Escobedo, Valdés Cuervo, Reyes Mendoza, \& Carlos Martínez, 2010; Cázarez \& Duarte, 2019). 
En cuanto a la validez del constructo, conforme al análisis factorial confirmatorio, los resultados obtenidos en la primera y segunda prueba arrojaron que el modelo de los tres factores no presentó un buen ajuste tal como sucedió con Pillati et al. (2012) en la primera prueba, que en el caso de ellos, para la búsqueda de un mejor ajuste, bastó con revisar los residuos de la matriz de la varianza y correlación, para esta investigación el procedimiento no fue suficiente, al igual que Hakspiel (2016), se procedió a mejorar las preguntas, dado que el análisis factorial confirmatorio arrojó que existen ítems que se relacionan entre grupos, por ejemplo las preguntas Q13 y Q30; Q2 y Q29; y Q8 y Q17, guardan relación entre ellas permitiendo unificar criterios, lo que lleva a reevaluar la pertenencia de cada pregunta a su respectivo grupo (ver tablas I, II y III).

Por otro lado, para efectividad en los resultados y mejora en la calidad de la evaluación del instrumento en cuanto a validez, se debe considerar trabajar con aumentar el tamaño de la muestra de los participantes como mínimo a doscientos, tanto para la prueba piloto, como la prueba definitiva. Esto debido a que al ser menor que este valor, pierden estabilidad los resultados, tal como lo menciona Veliecer (1998) y como lo sustenta el análisis factorial confirmatorio del presente estudio, aunque existen investigaciones en educación que elaboraron sus cuestionarios con muestras menores a doscientos, estas se construyeron basados en otros cuestionarios ya validados como el caso de Hakspiel et al. (2016) y Lacave et al. (2015). Sin embargo, existen estudios que a pesar de estar fundamentados en otros constructos, como Hora, Monterior y Arica (2010) y Cupé y García (2015), estos prefieren sujetarse a las recomendaciones de Lloret (2014), es decir, trabajar con muestras mayores a doscientas observaciones.

También se intentó recurrir a la práctica de modificación de índices para el modelo a efectos de validación, pero este procedimiento lleva a que pierda valor teórico el cuestionario, por alcanzar un mejor ajuste estadístico tal como lo menciona Pérez (2013), aunque existen autores que recurren a este procedimiento, como es el caso de Mérida (2015). Esto sería forzar la investigación a la validación del cuestionario para cumplir con el propósito o lo esperado por el estudio.

Por otra parte, el análisis descriptivo de la información evidenció la inclinación que tienen algunos docentes al contestar ciertas preguntas del cuestionario (ver tablas VII, IX y X) creando una tendencia, lo que lleva a hacer ajustes en las preguntas, con el fin de mejorar los resultados en la validación del constructo, tal como lo menciona Hernández et al. (2014). 
Los resultados arrojados por este estudio, se consolidan como una guía para futuras investigaciones interesadas en la búsqueda de un constructo, que además de ser confiable, como es el caso de esta investigación, logre ser validado con la finalidad de medir la acción pedagógica del docente, las estrategias y el ambiente donde se desarrollan estas estrategias en los grados de transición y primaria de las instituciones públicas del Departamento del Atlántico en Colombia, y a su vez facilite el desarrollo profesional puesto que son pocas las investigaciones sobre cuestionarios en educación tal como lo expresan Lacave et al. (2015).

\section{REFERENCIAS}

AGUERRONDO, I. (2009). La escuela inteligente en el marco de la gestión del conocimiento. Innovación Educativa, 9(47), 33-43. https://doi.org/10.24109/2176-6681.rbep.91i227.

CASCAES DA SILVA, F., GONÇALVES, E., VALDIVIA, B., GRAZIELE, G., SILVA, T., SOLEMAN, S., \& DA SILVA, R. (2015). Estimadores de consistencia interna en las investigaciones en salud: El uso del coeficiente alfa. Revista Peruana de Medicina $\begin{array}{lllll}\text { Experimental } \quad \text { S } & \text { Salud } & \text { Publica, } & \text { 32(1), } & \text { 138. }\end{array}$ https://doi.org/10.17843/rpmesp.2015.321.1585.

CÁZAREZ, C. R. C., \& DUARTE, G. A. L. (2019). Análisis factorial de un modelo de socialización y confianza en la dependencia de Internet en estudiantes de secundaria. Revista Electrónica de Investigación $\quad$ Educativa, $21(1), 13$. https://doi.org/10.24320/redie.2019.21.e25.2112.

COHEN, J., COHEN, P., WEST, S. G., \& AIKEN, L. S. (2002). Applied Multiple Regression/Correlation Analysis for the Behavioral Sciences (Edición: 3). Mahwah, N.J: Routledge.

CUPÉ-ARAUJO, A. C., \& GARCÍA-RUPAYA, C. R. (2015). Conocimientos de los padres sobre la salud bucal de niños preescolares: Desarrollo y validación de un instrumento. Revista Estomatológica Herediana, 25(2), 112-121. https://doi.org/10.20453/reh.2015.2457.

ESCOBEDO PORTILLO, M. T., HERNÁNDEZ GÓMEZ, J. A., ESTEBANÉ ORTEGA, V., \& MARTÍNEZ MORENO, G. (2016). Modelos de ecuaciones estructurales: Características, fases, construcción, aplicación y resultados. Ciencia \&amp; trabajo, 18(55), 16-22. https://doi.org/10.4067/S0718-24492016000100004.

HAKSPIEL-PLATA, M. C., NIÑO-BAUTISTA, L., VELAZCO-RANGEL, C. M., ARAGÓNBORRÉ, D., \& PINEDA-RODRÍGUEZ, L. P. (2016). Diseño y validación de encuesta sobre motivación de docentes frente a procesos de educación para la sexualidad. Universidad y Salud, 18(1), 46-57. https://doi.org/10.22267/rus.161801.18.

HERNÁNDEZ, R., FERNÁNDEZ, C., \& BAPTISTA, P. (2014). Metodología de la investigación (Sexta). 
HERRERO, J. (2010). El análisis factorial confirmatorio en el estudio de la estructura y estabilidad de los instrumentos de evaluación: Un ejemplo con el cuestionario de autoestima CA-14. Psychosocial Intervention, 19(3), 289-300. https://doi.org/10.5093/in2010v19n3a9.

HORA, H. R. M. DA, MONTEIRO, G. T. R., \& ARICA, J. (2010). Confiabilidade em Questionários para Qualidade: Um Estudo com o Coeficiente Alfa de Cronbach. Produto \& Produção, 11(2). https://doi.org/10.22456/1983-8026.9321.

LACAVE, C., MOLINA, A. I., FERNÁNDEZ, M., \& REDONDO, M. Á. (2015, julio 8). Análisis de la fiabilidad y validez de un cuestionario docente. 136-143. Recuperado de https://upcommons.upc.edu/handle/2117/76844.

LIZARBE, M., GUILLÉN-, F., AGUINAGA, I., \& CANGA, N. (2016). Validación del Cuestionario de Orientación a la Vida (OLQ-13) de Antonovsky en una muestra de estudiantes universitarios en Navarra. Anales del Sistema Sanitario de Navarra, 39(2), 237-248. Recuperado de http://scielo.isciii.es/scielo.php?script=sci_abstract\&pid=S1137-

$66272016000200006 \&$ Ing=es\&nrm=iso\&tIng=es.

LLORET, S., FERRERES, A., HERNÁNDEZ, A., \& TOMÁS, I. (2014). El Análisis Factorial Exploratorio de los Ítems: Una guía práctica, revisada y actualizada. Anales de Psicología, 30(3), 1151-1169. https://doi.org/10.6018/analesps.30.3.199361.

MAGAÑA, D. E. M., VÁSQUEZ, N. A., \& AGUILAR, J. M. V. (2017). Análisis factorial confirmatorio para medir las limitantes percibidas en el pregrado para el desarrollo de actividades de investigación. Nova Scientia, 9(18), 515-536. https://doi.org/10.21640/ns.v9i18.838.

MÉRIDA, R., SERRANO, A., \& TABERNERO, C. (2015). Diseño y validación de un cuestionario para la evaluación de la autoestima en la infancia. Revista de Investigación Educativa, 33(1), 149-162. https://doi.org/10.6018/rie.33.1.182391.

PÉREZ, E., MEDRANO, L. A., \& ROSAS, J. S. (2013). El Path Analysis: Conceptos básicos y ejemplos de aplicación. Revista Argentina de Ciencias del Comportamiento, 52-66. https://doi.org/10.32348/1852.4206.v5.n1.5160.

PÉREZ LÓPEZ, C. (2004). Técnicas de analisís multivariante de datos: Aplicaciones con SPSS (Edición: 1). Madrid etc.: ALHAMBRA.

PILATTI, A., GODOY, J., \& BRUSSINO, S. (2012). Análisis factorial confirmatorio del cuestionario de expectativas hacia el alcohol para adolescentes (CEA-A). Acta Colombiana de Psicología, 15(2), 11-20. Recuperado de http://www.scielo.org.co/scielo.php?script=sci_abstract\&pid=S0123-

$91552012000200002 \& \operatorname{lng}=$ en\&nrm=iso\&tlng=es.

RIVILLA, A. M. M. (2015). Innovación de la educación y de la docencia. Recuperado de https://dialnet.unirioja.es/servlet/libro?codigo $=579024$.

SÁNCHEZ ESCOBEDO, P. A., VALdÉS CUERVO, Á. A., REYES MENDOZA, N. M., \& CARLOS MARTÍNEZ, E. A. (2010). Participación de padres de estudiantes de educación 
primaria en la educación de sus hijos en méxico. Liberabit, 16(1), 71-80. Recuperado de http://www.scielo.org.pe/scielo.php?script=sci_abstract\&pid=S1729-

48272010000100008\&lng=es\&nrm=iso\&tIng=es.

SCHMIDT, D. R. C., \& DANTAS, R. A. S. (2011). Análise da validade e confiabilidade da versão adaptada para o português do Questionário de Senso de Coerência de Antonovsky, entre profissionais de enfermagem. Revista Latino-Americana de Enfermagem, 19(1), $42-49$. https://doi.org/10.1590/S0104-11692011000100007.

VELICER, W. F., \& FAVA, J. L. (1998). Affects of variable and subject sampling on factor pattern recovery. Psychological Methods, 3(2), 231-251. https://doi.org/10.1037/1082989X.3.2.231.

ZANTEN, A. VAN, NÓVOA, A., POPKEWITZ, T. S., BOOTE, D., LELIS, I., MAROY, C., ... KARSENTI, T. (2014). Ofício de professor: História, perspectivas e desafios internacionais (Edição: 6a). Petrópolis, RJ: Editora Vozes.

Artigo recebido em: 04 de novembro de 2019 Aceito para publicação em: 11 de janeiro de 2020 